九州大学学術情報リポジトリ

Kyushu University Institutional Repository

\title{
Self-similarity of binary quasiperiodic sequences
}

Odagaki, Takashi

Department of Physics, Kyushu Univevrsity | Department of Arts and Sciences, Kyoto Institute of Technology

Kaneko, Masanobu

Department of Arts and Sciences, Kyoto Institute of Technology

http://hdl. hand le. net/2324/20440

出版情報: Journal of Physics. A, Mathematical and general. 27 (5), pp.1683-1690，1994-03-07. IOP Publishing

バージョン :

権利関係 : 


\title{
Self-similarity of binary quasiperiodic sequences
}

\author{
T Odagaki† and M Kanekoł§ 9 \\ † Department of Physics, Kyushu University, Fukuoka 812, Japan \\ $\ddagger$ Department of Liberal Arts and Sciences, Kyoto Institute of Technology, Matsugasaki, \\ Kyoto 606, Japan
}

Received 2 June 1993, in final form 15 November 1993

\begin{abstract}
Self-similarity in binary quasiperiodic sequences generated by a projection method is shown to exist when and only when it is associated with quadratic irrational numbers and the explicit self-similarity transformation for an arbitrary quadratic number is obtained. The self-similarity transformation is shown not to be reducible to the simplest form for a class of quadratic numbers.
\end{abstract}

\section{Introduction}

Quasiperiodic systems lack any translational symmetry yet they are not random. The properties of a quasiperiodic system are considered to be fundamentally different from two extremes, regular crystals and random systems, since the geometrical structure plays a pivotal role in determining them. For example, the Bloch theorem and the van Hove singularities in the density of states for regular crystals follow from the translational symmetry. In elucidating the physical properties of quasiperiodic crystals, it is crucial to have clear knowledge of their geometrical structure and symmetry [1-6]. Even for onedimensional chains, however, there are only a few systems whose geometrical structure (self-similarity) is known well, despite extensive work over the past several years [7]. Among them are the Fibonacci chain and its relatives [3].

In this paper we consider quasiperiodic sequences of two components produced by a projection method with one parameter $\alpha$ and study the self-similarity of the sequences. In section 2 we prove that the necessary and sufficient conditions for a sequence to be selfsimilar is that $\alpha$ is a quadratic number. It is rather trivial to show that this condition is necessary for a sequence to be self-similar. Thus the main point of the discussion is to prove that the condition is also sufficient for self-similarity. To this end, we obtain the explicit selfsimilarity transformation of the quasiperiodic sequence for an arbitrary quadratic number, exploiting the continued-fraction expansion of an irrational number which has been utilized in the discussion of quasiperiodic systems $[1,8,9]$. We discuss the reduction of the selfsimilarity transformation in section 3 and show that the transformation cannot be reduced to its simplest form for a class of quadratic numbers. To illustrate an example of a non-selfsimilar quasi periodic sequence, we briefly discuss in section 4 the band structure of a tight binding electron on a one-dimensional chain. Concluding remarks are made in section 5 .

$\S$ Research fellow of the Alexander von Humboldt Foundation.

I Current address: Mathematisches Institut, Universität zu Köln, Weyertal 86-90, 50931 Köln, Germany. 


\section{The self-similarity transformation}

We consider a quasiperiodic sequence of 0 and 1 given by

$$
F(\alpha)=\left\{F_{n}(\alpha)\right\} \quad(n \geqslant 1)
$$

with

$$
F_{n}(\alpha)=[(n+1) \alpha]-[n \alpha]
$$

where $[x]$ denotes the integer part of $x, n=1,2,3, \ldots$ and $\alpha$ is a real parameter in $(0,1)$ characterizing the sequence. It should be emphasized that the following argument holds for any system isomorphic to $F(\alpha)$. When $\alpha$ is a (reduced) rational number $a / b(a<b)$, then the sequence is periodic, and we denote the periodic unit by $\Pi(a / b)$ which consists of $a 1$ $\mathrm{s}$ and $b-a$ os.

We define an inflation rule for sequence (1) as two simultaneous transformations for two units of 0 and 1 :

$$
\begin{aligned}
& S(0,1) \rightarrow S^{\prime}(0,1) \\
& T(0,1) \rightarrow T^{\prime}(0,1)
\end{aligned}
$$

where $S(0,1)$ and $T(0,1)$, units consisting of $k_{s} 0 \mathrm{~s}, l_{s} 1 \mathrm{~s}$ and $k_{t} 0 \mathrm{~s}, l_{t} 1 \mathrm{~s}$, respectively, cover the entire $F(\alpha)$. The numbers of 0 s and $1 \mathrm{~s}$ in $S^{\prime}(0,1), k_{s}^{\prime}, l_{s}^{\prime}$, and those in $T^{\prime}(0,1), k_{t}^{\prime}, l_{t}^{\prime}$, are assumed to satisfy $k_{s}^{\prime}+l_{s}^{\prime} \geqslant k_{s}+l_{s}$ and $k_{t}^{\prime}+l_{t}^{\prime} \geqslant k_{t}+l_{t}$. When $F(\alpha)$ is invariant under transformation (3), the sequence is called self-similar. For a self-similar sequence $F(\alpha)$, the ratio of the numbers of $0 \mathrm{~s}$ and $1 \mathrm{~s}$ in the system must be unchanged when the transformation is applied, and hence $\alpha$ must be a quadratic number determined by

$$
\begin{aligned}
{\left[( k _ { s } + l _ { s } ) \left(k_{t}^{\prime}+\right.\right.} & \left.\left.l_{t}^{\prime}\right)-\left(k_{t}+l_{t}\right)\left(k_{s}^{\prime}+l_{s}^{\prime}\right)\right] \alpha^{2} \\
& +\left[\left(k_{t}+l_{t}\right) l_{s}^{\prime}-\left(k_{t}^{\prime}+l_{t}^{\prime}\right) l_{s}+\left(k_{s}^{\prime}+l_{s}^{\prime}\right) l_{t}-\left(k_{s}+l_{s}\right) l_{t}^{\prime}\right] \alpha+l_{s} l_{t}^{\prime}-l_{t} l_{s}^{\prime}=0 .
\end{aligned}
$$

Thus, it is a rather trivial statement that parameter $\alpha$ of a self-similar quasiperiodic chain must be a quadratic number. Taking the contraposition, we can conclude that $F(\alpha)$ for $\alpha$ other than quadratic numbers does not have any self-similarity in the sense of equation (3). In the following, we show that the converse is also true, namely that the sequence $F(\alpha)$ for any quadratic number has self-similarity.

In order to find explicitly the self-similarity transformation of $F(\alpha)$ for an arbitrary quadratic number $\alpha$, we use the continued-fraction expansion of $\alpha$, which is known to be periodic beyond a certain level [10]†. We write it as

$$
\begin{aligned}
& \alpha=\frac{1}{k_{i}+} \frac{1}{k_{2}+} \cdots \frac{1}{k_{n-1}+\theta} \\
& \theta=\frac{1}{h_{1}+} \frac{1}{h_{2}+} \cdots \frac{1}{h_{m-1}+\theta} .
\end{aligned}
$$

\footnotetext{
$\uparrow$ Real numbers can be classified into algebraic and transcendental numbers, and the former are further classified according to the degree of the algebraic equation which they satisfy. A rational number is thus considered to be an algebraic number of degree one. An algebraic number of degree two is called a quadratic number, and is classified as a reduced or a non-reduced number: a quadratic number is called reduced when it is larger than unity and its conjugate is in $(-1,0)$; when a quadratic number is in $(0,1)$ and its conjugate is not in $(0,1)$ we call it a quasi-reduced quadratic number.
} 
The periodic part $\theta$ is the inverse of a reduced quadratic number $\theta \in(0,1)$ and its conjugate $\bar{\theta}<-1$ ). We denote the $i$ th approximant of $\alpha$ by $p_{i} / q_{i}$,

$$
\frac{p_{i}}{q_{i}}=\frac{1}{k_{1}+} \frac{1}{k_{2}+} \ldots \frac{1}{k_{i-1}} \quad i=2, \ldots, n
$$

and the $i$ th approximant of $\theta$ by $r_{i} / s_{i}$,

$$
\frac{r_{i}}{s_{i}}=\frac{1}{h_{1}+} \frac{1}{h_{2}+} \ldots \frac{1}{h_{i-1}} \quad i=2, \ldots, m
$$

$\left(p_{1} / q_{1}=r_{1} / s_{1}=0 / 1\right)$. Since $\theta$ is the stable fixed point of a modular transformation

$$
\gamma(\theta)=\frac{p \theta+q}{r \theta+s}
$$

with $p s-q r=1$ (we choose this parity since, when $m$ is odd, $p=r_{m}, q=r_{m-1}, r=$ $s_{m}, s=s_{m-1}$ and, when $m$ is even, $p=r_{m} s_{m-1}+r_{m-1}^{2}, q=r_{m}\left(s_{m}+r_{m-1}\right), r=$ $\left.s_{m-1}\left(s_{m}+r_{m-1}\right), s=s_{m}^{2}+r_{m} s_{m-1}\right), \alpha$ is the stable fixed point of a modular transformation,

$$
\delta(\alpha)=\frac{p_{n-1} \gamma[\theta(\alpha)]+p_{n}}{q_{n-1} \gamma[\theta(\alpha)]+q_{n}} \equiv \frac{A \alpha+B}{C \alpha+D} .
$$

Here,

$$
\theta(\alpha)=\frac{p_{n}-\alpha q_{n}}{\alpha q_{n-1}-p_{n-1}}
$$

and $A B-C D=p s-q r=1$ is shown to hold. In fact $A, B, C$ and $D$ are written as

$$
\begin{aligned}
& A=p_{n}\left(s q_{n-1}-r q_{n}\right)+p_{n-1}\left(q q_{n-1}-p q_{n}\right) \\
& B=p_{n}\left(r p_{n}-s p_{n-1}\right)+p_{n-1}\left(p p_{n}-q p_{n-1}\right) \\
& C=q_{n}\left(s q_{n-1}-r q_{n}\right)+q_{n-1}\left(q q_{n-1}-p q_{n}\right) \\
& D=q_{n}\left(r p_{n}-s p_{n-1}\right)+q_{n-1}\left(p p_{n}-q p_{n-1}\right) .
\end{aligned}
$$

The flow of the fixed point iteration

$$
\alpha^{\prime}=\delta(\alpha)
$$

can be easily analysed. We find:

(1) The complex conjugate $\bar{\alpha}$ of $\alpha$ is the unstable fixed point of equation (14), and it is the stable fixed point of $\bar{\alpha}^{\prime}=(-D \bar{\alpha}+B) /(C \bar{\alpha}-A)$.

(2) The convergence region $\Gamma_{\alpha}$ of equation (14) is given by $\Gamma_{\alpha}=(-\infty, \bar{\alpha})$ when $C>0$ and $n$ is odd or when $C<0$ and $n$ is even and $\Gamma_{\alpha}=(\bar{\alpha}, \infty)$ when $C>0$ and $n$ is even or when $C<0$ and $n$ is odd. 
We consider a series of transformtions

$$
\frac{p_{n}}{q_{n}} \rightarrow \delta\left(\frac{p_{n}}{q_{n}}\right) \rightarrow \delta^{2}\left(\frac{p_{n}}{q_{n}}\right) \rightarrow \delta^{3}\left(\frac{p_{n}}{q_{n}}\right) \rightarrow \ldots
$$

which can be shown to converge to $\alpha$ monotonically. Using the fact that $p_{n}=$ $k_{n-1} p_{n-1}+p_{n-2}, q_{n}=k_{n-1} q_{n-1}+q_{n-2}$ and $p_{n} q_{n-1}-q_{n} p_{n-1}=(-1)^{n}$, we can prove that $p_{n-2}^{\prime} / q_{n-2}^{\prime} \equiv\left(p_{n}-p_{n-1}\right) /\left(q_{n}-q_{n-1}\right), p_{n} / q_{n}$ and $p_{n-1} / q_{n-1}$ form a Farey triplet $\dagger$, which are all in the converging region, $\Gamma_{\alpha}$. Therefore, the periodic unit $\Pi\left(p_{n} / q_{n}\right)$ for $F\left(p_{n} / q_{n}\right)$ is obtained by putting $\Pi\left(p_{n-1} / q_{n-1}\right)$ and $\Pi\left(p_{n-2}^{\prime} / q_{n-2}^{\prime}\right)$ side by side:

$$
\Pi\left(\frac{p_{n}}{q_{n}}\right)= \begin{cases}\Pi\left(\frac{p_{n-1}}{q_{n-1}}\right) \Pi\left(\frac{p_{n-2}^{\prime}}{q_{n-2}^{\prime}}\right) & \text { when } n \text { is even } \\ \Pi\left(\frac{p_{n-2}^{\prime}}{q_{n-2}^{\prime}}\right) \Pi\left(\frac{p_{n-1}}{q_{n-1}}\right) & \text { when } n \text { is odd. }\end{cases}
$$

When $p_{n-2}^{\prime} / q_{n-2}^{\prime}=1$ for even $n$ or $p_{n-1} / q_{n-1}=1$ for odd $n$, equation (16) must be considered as

$$
\Pi\left(\frac{p_{n}}{q_{n}}\right)= \begin{cases}1 \Pi\left(\frac{p_{n-1}}{q_{n-1}}\right) & \text { when } n \text { is even } \\ 1 \Pi\left(\frac{p_{n-2}^{\prime}}{q_{n-2}^{\prime}}\right) & \text { when } n \text { is odd. }\end{cases}
$$

Note, however, that this happens only when $n=2, k_{1}=2$ or $n=4, k_{1}=k_{3}=1$ for even $n$ or $n=3, k_{1}=1$ for odd $n$. Since

$$
\begin{aligned}
& \delta\left(\frac{p_{n-2}^{\prime}}{q_{n-2}^{\prime}}\right)=\frac{p_{n}(s-r)+p_{n-1}(q-p)}{q_{n}(s-r)+q_{n-1}(q-p)} \\
& \delta\left(\frac{p_{n}}{q_{n}}\right)=\frac{p_{n} s+p_{n-1} q}{q_{n} s+q_{n-1} q} \\
& \delta\left(\frac{p_{n-1}}{q_{n-1}}\right)=\frac{p_{n} r+p_{n-1} p}{q_{n} r+q_{n-1} p}
\end{aligned}
$$

are also a Farey triplet, $\Pi\left[\delta\left(p_{n} / q_{n}\right)\right]$ is given in a similar manner to equation (16) with $\Pi\left[\delta\left(p_{n-1} / q_{n-1}\right)\right]$ and $\Pi\left[\delta\left(p_{n-2}^{\prime} / q_{n-2}^{\prime}\right)\right]$, which in turn are written as a product of $\Pi\left(p_{n-1} / q_{n-1}\right)$ and $\Pi\left(p_{n-1}^{\prime} / q_{n-2}^{\prime}\right)$ in the order determined by $p, q, r, s$, i.e. $\theta$. Therefore, for $i=1,2,3, \ldots, \Pi\left[\delta^{(i)}\left(p_{n} / q_{n}\right)\right]$ is obtained from $\Pi\left[\delta^{(i-1)}\left(p_{n} / q_{n}\right)\right]$ by the inflation

$$
\begin{aligned}
& \Pi\left(p_{n-1} / q_{n-1}\right) \rightarrow \Pi\left[\delta\left(p_{n-1} / q_{n-1}\right)\right] \\
& \Pi\left(p_{n-2}^{\prime} / q_{n-2}^{\prime}\right) \rightarrow \Pi\left[\delta\left(p_{n-2}^{\prime} / q_{n-2}^{\prime}\right)\right] .
\end{aligned}
$$

When $p_{n-2}^{\prime} / q_{n-2}^{\prime}=1$ for even $n$ or $p_{n-1} / q_{n-1}=1$ for odd $n$, we have to take the transformation for $\Pi(1)$ in equation $(21)$ as

$$
\Pi(1) \rightarrow \bar{\Pi}[\delta(1)]
$$

where $\bar{\Pi}(\ldots)$ denotes the same sequence as $\Pi(.$.$) except for 10$ at the right end being changed to 01 . It should be noted that the same modification applies in the following. As the inflation (21) holds at the fixed point of the modular transformation $\delta(\alpha)$, it is the self-similarity transformation of $F(\alpha)$ for the quadratic number $\alpha$ satisfying $\alpha=\delta(\alpha)$.

$\dagger$ The Farey series of order $m$ is the ascending series of irreducible fractions in $(0,1)$ whose denominators do not exceed $m(0 \equiv 0 / 1$ and $1 \equiv 1 / 1)$. A Farey triplet is a set of three successive terms in a Farey series. 


\section{Reduction of the self-similarity transformation}

We can define $p_{n-2}^{\prime \prime}$ and $q_{n-2}^{\prime \prime}$ by

$$
\frac{p_{n-2}^{\prime}}{q_{n-2}^{\prime}}=\frac{\left(k_{n-1}-1\right) p_{n-1}+p_{n-2}}{\left(k_{n-1}-1\right) q_{n-1}+q_{n-2}}=\frac{p_{n-1}+p_{n-2}^{\prime \prime}}{q_{n-1}+q_{n-2}^{\prime \prime}}
$$

and thus $\Pi\left(p_{n-2}^{\prime} / q_{n-2}^{\prime}\right)$ can be written in terms of $\Pi\left(p_{n-1} / q_{n-1}\right)$ and $\Pi\left(p_{n-2}^{\prime \prime} / q_{n-2}^{\prime \prime}\right)$. Consequently, if $p_{n-2}^{\prime \prime} / q_{n-2}^{\prime \prime}$ is in $\Gamma_{\alpha}$, the inflation (21) can be reducible to

$$
\begin{aligned}
& \Pi\left(p_{n-1} / q_{n-1}\right) \rightarrow \Pi\left[\delta\left(p_{n-1} / q_{n-1}\right)\right] \\
& \Pi\left(p_{n-2}^{\prime \prime} / q_{n-2}^{\prime \prime}\right) \rightarrow \Pi\left[\delta\left(p_{n-2}^{\prime \prime} / q_{n-2}^{\prime \prime}\right)\right] .
\end{aligned}
$$

One can repeat this process to reach

$$
\begin{aligned}
& \Pi\left(p_{n-1} / q_{n-1}\right) \rightarrow \Pi\left[\delta\left(p_{n-1} / q_{n-1}\right)\right] \\
& \Pi\left(p_{n-2} / q_{n-2}\right) \rightarrow \Pi\left[\delta\left(p_{n-2} / q_{n-2}\right)\right]
\end{aligned}
$$

as long as $p_{n-2} / q_{n-2}$ is in $\Gamma_{\alpha}$. Furthermore, noting that

$$
\frac{p_{n-1}}{q_{n-1}}=\frac{k_{n-2} p_{n-2}+p_{n-3}}{k_{n-2} q_{n-2}+q_{n-3}}
$$

we can reduce the inflation rule when $p_{n-3} / q_{n-3}$ is in $\Gamma_{\alpha}$. Since $\Gamma_{\alpha}$ for quasi-reduced quadratic numbers [10] contains $(0,1)$, the inflation rule can be reducible to

$$
\begin{aligned}
& \Pi(0)=0 \rightarrow \Pi[\delta(0)] \\
& \Pi(1)=1 \rightarrow \bar{\Pi}[\delta(1)] .
\end{aligned}
$$

An alternative proof for this inflation rule is given elsewhere [11].

For non-quasi-reduced quadratic numbers, the reduction to the rule (27) is not possible because their conjugate is in $(0,1)$ and the reduction cannot go beyond the converging region $\Gamma_{\alpha}$ given in section 2. As an illustration, let us consider

$$
\alpha=\frac{16-2 \sqrt{2}}{31}=\frac{1}{2+2+\theta} \frac{1}{1+4+\theta}
$$

thus

$$
\gamma(\theta)=\frac{\theta+4}{\theta+5} \quad \delta(\alpha)=\frac{13 \alpha-8}{31 \alpha-19} .
$$

The inflation rule corresponding to equation (21) is

$$
\begin{aligned}
& \Pi(1 / 2) \rightarrow \Pi[\delta(1 / 2)]=\Pi(3 / 7) \\
& \Pi(1 / 3) \rightarrow \Pi[\delta(1 / 3)]=\Pi(11 / 26) .
\end{aligned}
$$

Since $\Pi(1 / 3)=\Pi(0 / 1) \Pi(1 / 2)$, this is reducible to

$$
\begin{aligned}
& \Pi(0)=0 \rightarrow \Pi[\delta(0)]=\Pi(8 / 19)=0101001010100101010 \\
& \Pi(1 / 2)=10 \rightarrow \Pi[\delta(1 / 2)]=\Pi(3 / 7)=0101010 .
\end{aligned}
$$

This is the simplest (reduced) inflation rule for $F[(16-2 \sqrt{2}) / 31]$. 


\section{Non-self-similar sequences}

In order to see the properties of non-self-similar sequences, we consider as an example the band structure of a tight binding electron on a one-dimensional quasiperiodic chain where the site energy takes two different values $\epsilon_{0}$ and $\epsilon_{1}$ in the sequence of equation (1). It is known that the energy band is determined by the convergence of the product of two transfer matrices

$$
g_{0}=\left(\begin{array}{cc}
E-\epsilon_{0} & -1 \\
1 & 0
\end{array}\right) \quad g_{1}=\left(\begin{array}{cc}
E-\epsilon_{1} & -1 \\
1 & 0
\end{array}\right)
$$

Here, $E$ is the energy of the electron and the transfer energy is chosen as the scale of energy. We write the continued fraction expansion of $\alpha$ as

$$
\alpha=\frac{1}{k_{1}+} \frac{1}{k_{2}+} \ldots \frac{1}{k_{n-1}+} \ldots
$$

and define the $n$th approximant to $\alpha$ by $\left(p_{1} / q_{1}=0 / 1\right)$

$$
\frac{p_{n}}{q_{n}}=\frac{1}{k_{1}+} \frac{1}{k_{2}+} \ldots \frac{1}{k_{n-1}}
$$

We denote by $A_{n}$ the trace of the product of the matrices for the periodic unit of the $n$th approximant $\Pi\left(p_{n} / q_{n}\right)$. It is straightforward to show that

$$
\begin{aligned}
& A_{1}=\operatorname{Tr}\left(g_{0}\right) \\
& A_{2}=\operatorname{Tr}\left(g_{1} g_{0}\right) U_{k_{1}-2}\left(A_{1} / 2\right)-\operatorname{Tr}\left(g_{1}\right) U_{k_{1}-3}\left(A_{1} / 2\right) \\
& A_{n+1}=B_{n-1} U_{k_{n}-1}\left(A_{n} / 2\right)-A_{n-1} U_{k_{n}-2}\left(A_{n} / 2\right) \quad(n \geqslant 2)
\end{aligned}
$$

where

$$
\begin{aligned}
& B_{1}=\operatorname{Tr}\left(g_{1} g_{0}\right) U_{k_{1}-1}\left(A_{1} / 2\right)-\operatorname{Tr}\left(g_{1}\right) U_{k_{1}-2}\left(A_{1} / 2\right) \\
& B_{n}=B_{n-1} U_{k_{n}}\left(A_{n} / 2\right)-A_{n-1} U_{k_{n}-1}\left(A_{n} / 2\right) \quad(n \geqslant 2)
\end{aligned}
$$

and

$$
U_{m}(x)=\frac{\sin (m+1) \theta}{\sin \theta} \quad x=\cos \theta
$$

is the Chebyshev polynomial of the second kind [12]. Here, we have used the following generalized hyperinflation rule [6]: when $n$ is even

$$
\Pi\left(\frac{p_{n+1}}{q_{n+1}}\right)= \begin{cases}\Pi\left(\frac{p_{n-1}}{q_{n-1}}\right) \underbrace{\Pi\left(\frac{p_{n}}{q_{n}}\right) \ldots \Pi\left(\frac{p_{n}}{q_{n}}\right)}_{k_{n}} & p_{n} / q_{n} \neq 1 \\ \underbrace{1 \ldots 1}_{k_{n}} \Pi\left(\frac{p_{n-1}}{q_{n-1}}\right) & p_{n} / q_{n}=1\end{cases}
$$


and when $n$ is odd

$$
\Pi\left(\frac{p_{n+1}}{q_{n+1}}\right)= \begin{cases}\underbrace{\Pi\left(\frac{p_{n}}{q_{n}}\right) \ldots \Pi\left(\frac{p_{n}}{q_{n}}\right)}_{k_{n}} \Pi\left(\frac{p_{n-1}}{q_{n-1}}\right) & p_{n-1} / q_{n-1} \neq 1 \\ \underbrace{\Pi\left(\frac{p_{n}}{q_{n}}\right) \ldots \Pi\left(\frac{p_{n}}{q_{n}}\right)}_{k_{n}-1} 1 \Pi\left(\frac{p_{n}}{q_{n}}\right) & p_{n-1} / q_{n-1}=1 .\end{cases}
$$

The allowed energy band for the chain is determined by $\left|A_{n}\right| \leqslant 2$. We show in figure 1 the allowed energy region for

$$
\alpha=\frac{e^{2}-1}{e^{2}+1}=\frac{1}{1+3+5+} \frac{1}{5} .
$$

a transcendental number, when $\epsilon_{0}=0$ and $\epsilon_{1}=1$. It is clear that there is no self-similarity in the band structure. For the $n$th approximant crystal there are $q_{n}$ bands and, for the $(n+1)$ st approximant there are $q_{n+1}=k_{n} q_{n}+q_{n-1}$ bands; that is, besides each of $q_{n}$ bands in the $n$th approximant breaks up into $k_{n}$ bands, $q_{n-1}$ new bands appear. Thus, when one moves on to the higher order approximant, more bands appear than Azbel' [1] has predicted.

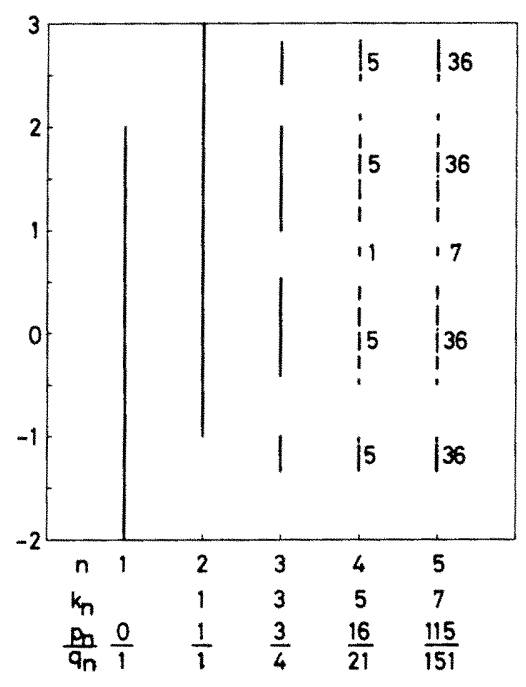

Figure 1. The allowed energy regions for a tight binding electron in approximant chains. Two site energies 0 and $I$ are placed in the order of the approximant to $F\left[\left(e^{2}-1\right) /\left(e^{2}+1\right)\right]$. For each approximant $p_{n} / q_{n}$, the allowed region consists of $q_{n}$ bands. For $p_{n+1} / q_{n+1}$, each band for $p_{n} / q_{n}$ splits into $k_{n}$ bands and, in addition to these, $q_{n-1}$ new bands appear. The numbers in the figure denote the number of bands in each bunch of the allowed region.

\section{Concluding remarks}

We have shown in this paper that there is a clear distinction between the quadratic algebraic numbers and other irrational numbers in self-similarity of one-parameter binary 
quasiperiodic chains and obtained the explicit algorithm to find the self-similarity transformation for a given quadratic number. Self-similarity in the form of equation (3) does not exist in the sequence except for quadratic irrational numbers. Self-similarity based on the inflation rule (27) exists only for the sequence corresponding to quasireduced quadratic numbers. For non-quasi-reduced quadratic numbers, the self-similarity transformation cannot be reduced to rule (27).

When $\alpha$ is a rational number/an algebraic number of degree one, there is a translational symmetry and in turn the Block or the Floquet theorem holds. Therefore, it is an intriguing problem to find if a general theorem exists in the properties of self-similar chains which corresponds to algebraic numbers of degree two and to investigate symmetries of quasiperiodic chains for other irrationals. In this connection, it should be noted that there has been an argument that the stability conditions for quasicrystals in two dimensions could be satisfied only for quadratic irrationalities [13]. As is well known [6, 14], many physical systems can be described in terms of unimodular transfer matrices, including an electric circuit and optical layers. Therefore, it will be feasible to test the present results by experiments.

\section{Acknowledgments}

This work was supported in part by a Grant-in-Aid for Scientific Research on Priority Areas from the Ministry of Education, and Science and Culture.

\section{References}

[1] Azbel' M Ya 1964 Zh. Eksp. Teor. Fiz. 46939 (1964 Sov. Phys,-JETP 19 634)

[2] Hofstadter D R 1976 Phys. Rev. B 142239

[3] Khomoto M, Kadanoff L P and Tang C 1983 Phys, Rev. Lett. 501870 Hawrylak P, Eliasson G and Quinn J J 1987 Phys, Rev. B 366501

Hashimoto Y, Niizeki K and Okabe Y 1992 J. Phys. A: Math. Gen. 255211

[4] Goda M and Kubo H 1990 J. Phys. Soc. Japan 583624

[5] Lunnon W F and Pleasants P A R 1987 J. Math. Pures Appl. 66217

[6] Iguchi K 1991 Phys. Rev. B 43 5915, 5919

[7] Steinhardt P J and Ostlund S (ed) 1987 The Physics of Quasicrystals (Singapore: World Scientific) Jaric M V (ed) 1988 Introduction to Quasicrystals (San Diego: Academic)

Fujiwara T and Ogawa T (ed) 1990 Quasicrystals (Berlin: Springer) Kuo K H and Ninomiya T (ed) 1991 Quasicrystals (Singapore: World Scientific)

[8] Suslov I M 1982 Zh. Eksp. Teor. Fiz. 831079 (1983 Sov. Phys.-JETP 56 612)

[9] Aubry S, Godrèche C and Luck J M 1988 J. Stat. Phys. 511033

[10] Hardy G H and Wright E M 1979 An Introduction to the Theory of Numbers (Oxford: Oxford University Press)

Baker A 1986 A Concise Introduction to the Theory of Numbers (Cambridge: Cambridge University Press)

[11] Kaneko M and Odagaki T 1993 J. Phys. Soc. Japan 621147

[12] Abramowitz M and Stegun 1 A 1965 Handbook of Mathematical Functions (New York: Dover) p 776 $(22.3 .16)$

[13] Levitov L S 1988 Europhys. Lett. 6517

[14] Odagaki T and Aoyama H 1988 Phys. Rev. Lett. 61 775; 1989 Phys. Rev. B 39475 